\title{
Mutually exclusive grounding for weakly supervised Non-negative Matrix Factorisation
}

\author{
Vincent Renkens, Hugo Van hamme \\ Department Electrical Engineering-ESAT, Katholieke Universiteit Leuven \\ Kasteelpark Arenberg 10, Bus 2441, B-3001 Leuven Belgium \\ \{vincent.renkens, hugo.vanhamme\}@esat.kuleuven.be
}

\begin{abstract}
Non-negative Matrix Factorisation (NMF) has been successfully applied for learning the meaning of a small set of vocal commands without any prior knowledge of the language. This kind of learning is useful if flexibility in terms of the acoustic and language model is required, for example in assistive technologies for dysarthric speakers because they do not comply with common models. Vocal commands are grounded through the addition of semantic labels that represent the action corresponding to the command. The Kullback Leibler Divergence (KLD) is used to evaluate the acoustic model. The KLD is optimal for Poisson distributed data making it an appropriate metric for the acoustic features because they are a count of acoustic events. The semantic labels are however activations, so a multinomial likelihood function seems more appropriate because they are mutually exclusive. In this paper a cost function to evaluate the semantic model based on the multinomial likelihood function is proposed that aims to better suit its distribution. To minimise the proposed cost function a new set of update rules and a new normalisation scheme are proposed.

Index Terms: vocabulary learning, human-computer interaction, speech recognition, non-negative matrix factorisation, machine learning
\end{abstract}

\section{Introduction}

Speech technology is often used as an interface to facilitate human-machine interaction. To allow an easy use of the interface for all users, flexibility in terms of acoustics, vocabulary and/or grammar is required from the spoken interface. It is in this respect that most command and control systems fall short. Traditionally, speech interfaces are equipped with a vocabulary and grammar [1] and a determined set of corresponding actions prior to deployment. However, in some applications determining the vocabulary and grammar is very expensive or simply not possible and the set of actions required from the interface may not be known in advance. Flexibility in terms of the vocal acoustics can be added by allowing the acoustic model to adjust to the speaker as in e.g. [2]. However when the speech of the user is very different from typical speech this approach is insufficient [3, 4].

In this paper a fully flexible interface is pursued where no assumptions are made about the user in terms of the vocal acoustics, vocabulary or grammar, nor are the actions preprogrammed. The system is trained by the end users making it maximally adjusted to them. The user can train the system by demonstrating each spoken command through an alternative interface. The spoken commands are then grounded based on semantic labels representing the corresponding action making it a weakly supervised technique. The term weakly supervised is used because only a semantic representation of the action is used as grounding information and not a transcription of the command. The language is thus acquired from the user's commands and demonstrations.

In prior work we have proposed a system for language acquisition based on NMF $[5,6]$. We have shown that this technique can acquire categorical concepts and that its results are comparable to other state-of-the-art techniques like e.g. techniques based on HMMs [7]. An application where categorical concepts need to be acquired is e.g. a home automation system where the TV can be controlled or the lights can be switched on or off.

NMF is used to find recurring acoustic patterns and couple them to semantic labels corresponding to the categorical concepts (called grounding information). This model is found by minimising some cost function. The cost function that has to be minimised is chosen based on the underlying distribution of the data [8]. In the past the Kullback-Leibler Divergence (KLD) was used to evaluate both the semantic and the acoustic model [6]. KLD is optimal for Poisson distributed data. For the acoustics assuming Independent Poisson distributed data is reasonable because Histogram of co-occurrence (HAC) features are used, which are counts of acoustic events [9]. However, the semantics are mutually exclusive semantic label activations, for these labels assuming independence is not valid any more. A multinomial distribution seems better suited here.

In this paper a cost function based on the multinomial distribution is proposed to evaluate the semantic model. Based on this cost function, a new set of update rules and a new normalisation scheme are proposed. The new updating scheme is compared to the updating scheme where KLD is used for both the semantics and the acoustics on three databases. The GRABO database contains recordings of users controlling a small robot, the PATCOR database contains recordings of a vocally guided card game called "Patience" and the DOMOTICA-4 database contains recordings of dysarthric speakers controlling a home automation system.

The remainder of the paper is organized as follows. In section 2 the system based on weakly supervised NMF is explained. In section 3 the new cost function is proposed and in section 4 the normalisation required by this new cost function is discussed. In section 5 the experimental setup is explained and the results are presented and discussed in section 6 . Finally some future work will be discussed in section 7 and some conclusions will be formulated in section 8 . 


\begin{tabular}{|c|c|c|c|c|c|}
\hline \multicolumn{3}{|c|}{$a_{1}$} & \multicolumn{2}{|c|}{$a_{2}$} & $a_{3}$ \\
\hline & & $b_{2}$ & $c_{1}$ & $c_{2}$ & \\
\hline$d_{1}$ & $d_{2}$ & & & & \\
\hline
\end{tabular}

Table 1: Dummy example of hierarchical structure of the semantic labels. $a, b, c$ and $d$ are different families.

\section{Language acquisition framework}

\subsection{Grounding the acoustics}

In this framework the acoustics are grounded through the addition of semantic labels. These labels correspond to concepts in the world of the system. For example the command: "open the door" could have the label "open" and "door". For each demonstration given to the robot a vector is created where each element of this vector corresponds to a label that can either be active (1) or not active (0).

All the labels are organised in a hierarchical structure [10]. One branch of the structure could for example correspond to manipulating doors. This branch would have sub-branches for all the possible doors and for opening and closing. Some of these labels are mutually exclusive, like opening and closing or which door is active (when the assumption is made that only one door can be opened or closed at a time). In this paper the labels that are mutually exclusive to each other will be called a family. A dummy example of a hierarchical structure can be seen in table 1. Every letter corresponds to a family and the subscripts are the indices in the family An example of a label vector can be seen in (1) where the highlighted labels in table 1 are activated.

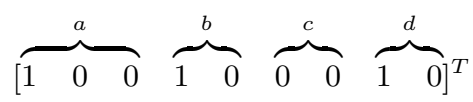

\subsection{Weakly supervised NMF}

In NMF a high dimensional, high rank $F \times N$ data matrix $\boldsymbol{V}$ is decomposed in two matrix factors of lower rank, the $F \times K$ dictionary matrix $\boldsymbol{W}$, which contains the latent components of the data, and the $K \times N$ activation matrix $\boldsymbol{H}$, which contains the activations of the latent components. Because the matrix factors are of lower rank, the decomposition is in general only an approximation:

$$
\boldsymbol{V} \approx \boldsymbol{W H}
$$

The training data matrix $\boldsymbol{V}^{\mathrm{trn}}$ is constructed by stacking the label vectors together with a vector representation of the vocal command. To represent the vocal commands Histogram of Acoustic Co-occurrences (HAC) vectors are used [9]. In the training phase this data matrix is decomposed similar to equation (2):

$$
\left[\begin{array}{l}
\boldsymbol{V}_{0}^{\mathrm{trn}} \\
\boldsymbol{V}_{1}^{\mathrm{trn}}
\end{array}\right] \approx\left[\begin{array}{l}
\boldsymbol{W}_{0} \\
\boldsymbol{W}_{1}
\end{array}\right] \boldsymbol{H}^{\mathrm{trn}}
$$

$\boldsymbol{V}_{\mathbf{0}}^{\text {trn }}$ is part of the training data matrix containing the label vectors, $\boldsymbol{V}_{\mathbf{1}}^{\text {trn }}$ is part of the training data matrix containing the HACvectors, $\boldsymbol{W}_{0}$ is the semantic dictionary and $\boldsymbol{W}_{1}$ is the acoustic dictionary. In the testing phase only the HAC-vectors $V_{1}^{\text {tst }}$ are available. It is first decomposed in its matrix factors, but now with a known dictionary:

$$
\boldsymbol{V}_{1}^{\mathrm{tst}} \approx \boldsymbol{W}_{1} \boldsymbol{H}^{\mathrm{tst}}
$$

The activations of the acoustic components $\boldsymbol{H}^{\text {tst }}$ and the semantic dictionary $\boldsymbol{W}_{0}$ are used to estimate the unknown semantic data by calculating the labelling matrix $\boldsymbol{A}$ :

$$
\boldsymbol{V}_{0}^{\text {tst }} \approx \boldsymbol{A}=\boldsymbol{W}_{0} \boldsymbol{H}^{\mathrm{tst}}
$$

The labelling matrix contains an estimation of the probabilities that a label is activated in the command. The command is classified by choosing the action with the highest estimated probability.

\section{Proposed cost function and updating rules}

The matrix factors are found by minimizing some cost function, which is a discrepancy measure between the model $\boldsymbol{W} \boldsymbol{H}$ and the data $\boldsymbol{V}$ [11]. In previous works KLD was used [6]. KLD is optimal for Poisson distributed data [8]. KLD is thus a good cost function for the acoustic data since the features are counts. However, the semantic labels are mutually exclusive semantic label activations. Using the KLD does not take this mutual exclusivity into account and it is thus suboptimal. Using a multinomial likelihood function does take this into account and is thus better suited for the semantic data. It is assumed that each normalised component of the dictionary corresponding to a family contains a probability distribution for its labels and the approximation is a mixture of these probability distributions with the normalised activations as mixture weights:

$$
\begin{aligned}
& D_{\text {mult }}\left(\boldsymbol{V}_{0}|| \boldsymbol{W}_{0} \boldsymbol{H}\right)=-\log P\left(\boldsymbol{V}_{0} \mid \boldsymbol{W}_{0} \boldsymbol{H}\right) \\
& =\sum_{n=1}^{N} \sum_{c=1}^{C} \sum_{f=1}^{\left|\mathcal{F}_{c}\right|}-V_{0, c}^{f n} \log \left(\sum_{k=1}^{K} \frac{W_{0, c}^{f k}}{\sum_{l=1}^{\left|\mathcal{F}_{c}\right|} W_{0, c}^{l k}} \frac{H^{k n}}{\sum_{l=1}^{K} H^{l n}}\right)
\end{aligned}
$$

Where $C$ is the number of families in the semantic data, $\boldsymbol{W}_{0, c}$ is the part of the semantic dictionary corresponding to family $c, \boldsymbol{V}_{0, c}$ is the part of the semantic data matrix corresponding to family $c$ and $\left|\mathcal{F}_{c}\right|$ is size of family $c$.

Notice that when this cost function is used it is assumed that every component contains information about every family. This is not the case. In the ideal case each component contains only one word and thus only contains information about one family. To solve this a "none of the above" label is added to the dictionary for every family. This label should be active if the component does not contain information about that family. To illustrate this, the semantics of the ideal components for the example shown in (1) are shown. This example contains three labels so there should be three active components:

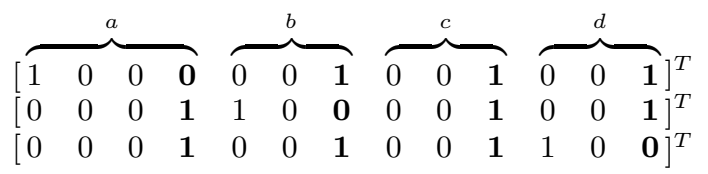

The labels in bold are the added labels. Notice that for these components the sum of the labels corresponding to a family is always equal to one. When these components are summed, the following label vector is obtained:

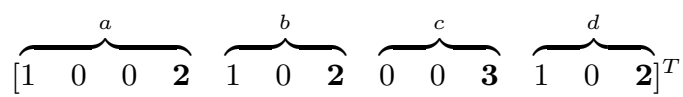


The bold labels will be added to the semantic data matrix. The value of the label can be calculated as the sum of all labels minus the sum of the labels corresponding to its family.

The update rules for the dictionaries for each family that minimise the proposed cost function are:

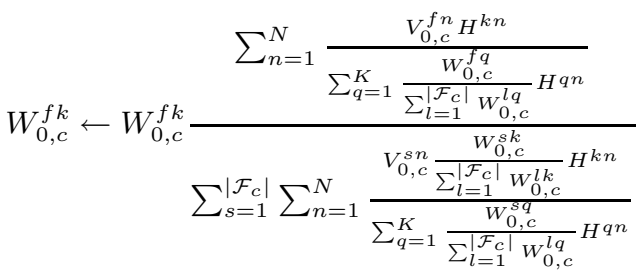

When this update rule is summed in the first dimension it always sums to 1 . This means that when this update rule is applied the elements in a dictionary component that correspond to the same family will automatically sum to 1 . The normalisation is thus no longer required and the update rule can be simplified to:

$$
W_{0, c}^{f k} \leftarrow W_{0, c}^{f k} \frac{\sum_{n=1}^{N} \frac{V_{0, c}^{f n}}{\left(W_{0, c} H\right)^{f n}} H^{k n}}{\sum_{s=1}^{\left|\mathcal{F}_{c}\right|} \sum_{n=1}^{N} W_{0, c}^{s k} \frac{V_{0, c}^{s n}}{\left(W_{0, c} H\right)^{s n}} H^{k n}}
$$

For the acoustic dictionary the update rule proposed in [11] for minimizing the KLD is used:

$$
W_{1}^{f k} \leftarrow W_{1}^{f k} \frac{\sum_{n=1}^{N} \frac{V_{1}^{f n}}{\left(W_{1} H\right)^{f n}} H^{k n}}{\sum_{n=1}^{N} H^{k n}}
$$

The update rule in (5) is similar to the update rule based on the KLD. If the dictionary would be normalised after applying the update rule in (6), the update rule in (5) would be obtained.

To update the activations $\boldsymbol{H}$ the total cost function must be minimised. This cost function is the sum of the cost functions for the semantic and acoustic part:

$$
D(\boldsymbol{V} \| \boldsymbol{W} \boldsymbol{H})=D_{\text {mult }}\left(\boldsymbol{V}_{0} \| \boldsymbol{W}_{0} \boldsymbol{H}\right)+D_{\mathrm{KLD}}\left(\boldsymbol{V}_{1} \| \boldsymbol{W}_{1} \boldsymbol{H}\right)
$$

Minimising this cost function leads to the following update rule for $\boldsymbol{H}$

$$
H^{k n} \leftarrow H^{k n} \frac{\sum_{c=1}^{C} \sum_{f=1}^{\left|\mathcal{F}_{c}\right|} \frac{V_{0, c}^{f n}}{\left(W_{0, c} H\right)^{f n}}+\sum_{f=1}^{F_{1}} \frac{V_{1}^{f n}}{\left(W_{1} H\right)^{f n}}}{\frac{\sum_{c=1}^{C} \sum_{f=1}^{\left|\mathcal{F}_{c}\right|} V_{0, c}^{f n}}{\sum_{q=1}^{K} H_{q n}}+\sum_{f=1}^{F_{1}} W^{f k}}
$$

$F_{1}$ is the number of acoustic labels.

\section{Normalisation}

Because there exists an infinite number of transformations of the dictionary and its activations that can generate an equivalent solution for equation (2), the solution is never unique [12]:

$$
\boldsymbol{V} \approx \boldsymbol{W} \boldsymbol{H}=(\boldsymbol{W} \boldsymbol{T})\left(\boldsymbol{T}^{-1} \boldsymbol{H}\right)
$$

Where $\boldsymbol{T}$ is an invertible matrix with a non-negative inverse, this could for example be a non-negative diagonal matrix, in this case the model is scaled. To alleviate this problem the solution is normalised to deal with the scaling ambiguity. This can be done by either normalising the columns of $\boldsymbol{W}$ or the rows of $\boldsymbol{H}$. In previous work it was chosen to normalise the columns of $\boldsymbol{W}$ to sum to 1 [13]. To keep the model to a correct scale the columns of $\boldsymbol{H}$ are scaled [13]. However when the multinomial cost function is used, the columns of $\boldsymbol{W}$ already have a fixed scale, they do not need to be normalised. The acoustic dictionary is thus normalised separately. Scaling the columns of $\boldsymbol{H}$ does not have any influence on the multinomial cost function. They can thus be scaled so only the acoustic approximation has the correct scale:

$$
m_{n}=\frac{\sum_{f=1}^{F_{1}} V_{1}^{f n}}{\sum_{k=1}^{K} H^{k n}}
$$

$m_{n}$ is the scale parameter for the $n^{\text {th }}$ column of $\boldsymbol{H}$.

\section{Experiments}

\subsection{Baseline system}

The baseline system used to compare the results uses the same framework as the proposed system but uses update rules based on the KLD for both the semantic and acoustic model. The columns of $\boldsymbol{W}$ are normalised and the factors for the columns of $\boldsymbol{H}$ are derived to minimise the KLD.

\subsection{Cross-validation}

Because a speaker dependent system is proposed, the amount of training data is limited. To get more significant results crossvalidation is used. First the data is divided into a number of blocks. The blocks are found by maximising the JensenShannon divergence between the blocks. Then training is done with a number of blocks, the rest of the blocks are used as training data. Each of these experiments is repeated five times, each time with a different subset of blocks. The average result of these 5 experiments will be reported.

\subsection{Databases}

The GRABO database is a database where users were asked to control a small robot. This robot can drive with different speeds, has an arm to grab and lift objects and a laser pointer is mounted on the arm. In total 11 users were recorded each uttering 36 different commands with 15 repetitions. Each user could translate the command in his or her own mother tongue and choose his or her own wordings. A typical command in this database is "quickly drive a little bit forward".

The PATCOR database [14] is a database with recorded sessions of a vocally guided card game called "Patience". This database is less controlled then the GRABO database because the commands are not intentionally repeated. The number of occurrences per word can vary greatly and some players used synonyms for certain cards. The amount of data per player varies because some games are longer then others.

The DOMOTICA-4 database contains recordings of impaired, dysarthric speakers controlling a home automation system. This database is a continuation of the DOMOTICA-2 database [14]. A typical command in this database is "Open the front door". This is the most challenging database because the dysarthric speech has greater variability making it harder to find patterns. 


\begin{tabular}{|c|c|c|c|c|}
\hline \# blocks & \multicolumn{2}{|c|}{ commands } & \multicolumn{2}{c|}{ labels } \\
\hline & mult & KLD & mult & KLD \\
\hline 1 & $\mathbf{0 . 9 5 9}$ & 0.594 & $\mathbf{0 . 9 8 7}$ & 0.730 \\
2 & $\mathbf{0 . 9 8 6}$ & 0.643 & $\mathbf{0 . 9 9 6}$ & 0.707 \\
3 & $\mathbf{0 . 9 8 8}$ & 0.666 & $\mathbf{0 . 9 9 6}$ & 0.704 \\
4 & $\mathbf{0 . 9 9 0}$ & 0.679 & $\mathbf{0 . 9 9 7}$ & 0.708 \\
5 & $\mathbf{0 . 9 9 3}$ & 0.692 & $\mathbf{0 . 9 9 7}$ & 0.715 \\
6 & $\mathbf{0 . 9 9 2}$ & 0.697 & $\mathbf{0 . 9 9 7}$ & 0.714 \\
7 & $\mathbf{0 . 9 9 3}$ & 0.709 & $\mathbf{0 . 9 9 8}$ & 0.724 \\
8 & $\mathbf{0 . 9 9 5}$ & 0.700 & $\mathbf{0 . 9 9 8}$ & 0.714 \\
9 & $\mathbf{0 . 9 9 4}$ & 0.715 & $\mathbf{0 . 9 9 8}$ & 0.726 \\
\hline
\end{tabular}

Table 2: Results for the Grabo database. The fraction of correct labels and correct commands are displayed in function of the number of blocks in the training set averaged over all speakers.

\begin{tabular}{|c|cccccccc|}
\hline \# blocks & 1 & 2 & 3 & 4 & 5 & 6 & 7 & 8 \\
\hline 1 & 0.29 & 0.13 & 0.09 & 0.14 & 0.30 & 0.28 & 0.51 & 0.28 \\
2 & 0.32 & 0.26 & 0.22 & 0.26 & 0.47 & 0.26 & 0.56 & 0.51 \\
3 & 0.40 & 0.34 & 0.29 & 0.46 & 0.58 & 0.42 & $\mathbf{0 . 5 9}$ & 0.63 \\
4 & 0.39 & 0.34 & 0.42 & 0.47 & 0.58 & 0.42 & $\mathbf{0 . 5 9}$ & 0.71 \\
5 & & 0.38 & 0.49 & & & 0.48 & & \\
6 & & 0.42 & & & & & & \\
\hline
\end{tabular}

(a)

\begin{tabular}{|c|c|c|c|c|c|c|c|c|}
\hline \# blocks & 1 & 2 & 3 & 4 & 5 & 6 & 7 & 8 \\
\hline 1 & 0.63 & 0.57 & 0.57 & 0.55 & 0.65 & 0.48 & 0.57 & 0.71 \\
\hline 2 & 0.67 & 0.71 & 0.61 & 0.57 & 0.76 & 0.50 & 0.57 & 0.80 \\
\hline 3 & 0.70 & 0.78 & 0.62 & 0.57 & 0.76 & 0.51 & 0.59 & 0.83 \\
\hline 4 & 0.72 & 0.79 & 0.62 & 0.59 & 0.81 & 0.51 & 0.59 & 0.84 \\
\hline 5 & & 0.81 & 0.62 & & & 0.51 & & \\
\hline 6 & & 0.82 & & & & & & \\
\hline
\end{tabular}

(b)

Table 3: Fraction of correct commands for the PATCOR database for each speaker in function of the number of blocks in the training set. Each column contains the results for one player. (a) Baseline system (b) Proposed system

\section{Results}

\subsection{Database: GRABO}

Both systems are tested on the GRABO database to recognise spoken commands given to a robot. The average fraction of correct labels and the fraction of commands that were completely correct are displayed in table 2 . The results can be averaged over all users because the content of the data is the same for all the users. The results show that the proposed system performs significantly better than the baseline system for all training set sizes, both in terms of correct labels and correct commands.

\subsection{Database: $P A T C O R$}

The fraction of correct commands in function of the number of blocks in the training set for all players in the PATCOR database are displayed in table 3. These results again show that the proposed system performs significantly better than the baseline system for all players.

\subsection{Database: DOMOTICA-4}

Table 4 shows the fraction of correct commands in function of the number of blocks in the training set for all users in the

\begin{tabular}{|c|ccccccc|}
\hline \# blocks & 1 & 2 & 3 & 4 & 5 & 6 & 7 \\
\hline 1 & 0.43 & 0.42 & 0.34 & 0.36 & 0.22 & 0.52 & 0.42 \\
2 & 0.54 & $\mathbf{0 . 6 1}$ & 0.47 & 0.51 & 0.30 & 0.65 & 0.55 \\
3 & 0.67 & $\mathbf{0 . 6 1}$ & 0.50 & 0.52 & 0.37 & 0.76 & 0.58 \\
4 & 0.70 & $\mathbf{0 . 7 9}$ & 0.63 & 0.64 & 0.42 & 0.80 & 0.64 \\
5 & 0.73 & $\mathbf{0 . 6 8}$ & 0.62 & & 0.41 & 0.83 & \\
6 & & & 0.66 & & $\mathbf{0 . 4 4}$ & 0.86 & \\
7 & & & 0.65 & & $\mathbf{0 . 4 5}$ & 0.86 & \\
8 & & & 0.67 & & $\mathbf{0 . 4 6}$ & $\mathbf{0 . 8 9}$ & \\
9 & & & 0.70 & & $\mathbf{0 . 5 4}$ & $\mathbf{0 . 8 8}$ & \\
\hline
\end{tabular}

(a)

\begin{tabular}{|c|ccccccc|}
\hline \# blocks & 1 & 2 & 3 & 4 & 5 & 6 & 7 \\
\hline 1 & $\mathbf{0 . 5 9}$ & $\mathbf{0 . 4 3}$ & $\mathbf{0 . 5 2}$ & $\mathbf{0 . 4 3}$ & $\mathbf{0 . 3 2}$ & $\mathbf{0 . 6 5}$ & $\mathbf{0 . 6 2}$ \\
2 & $\mathbf{0 . 7 5}$ & 0.54 & $\mathbf{0 . 6 5}$ & $\mathbf{0 . 6 4}$ & $\mathbf{0 . 4 0}$ & $\mathbf{0 . 8 0}$ & $\mathbf{0 . 7 5}$ \\
3 & $\mathbf{0 . 8 4}$ & 0.47 & $\mathbf{0 . 7 3}$ & $\mathbf{0 . 6 9}$ & $\mathbf{0 . 4 5}$ & $\mathbf{0 . 8 4}$ & $\mathbf{0 . 8 5}$ \\
4 & $\mathbf{0 . 8 8}$ & 0.64 & $\mathbf{0 . 7 6}$ & $\mathbf{0 . 8 0}$ & $\mathbf{0 . 4 3}$ & $\mathbf{0 . 8 7}$ & $\mathbf{0 . 8 7}$ \\
5 & $\mathbf{0 . 9 0}$ & 0.67 & $\mathbf{0 . 7 7}$ & & $\mathbf{0 . 4 2}$ & $\mathbf{0 . 8 9}$ & \\
6 & & & $\mathbf{0 . 7 9}$ & & 0.42 & $\mathbf{0 . 8 9}$ & \\
7 & & & $\mathbf{0 . 7 8}$ & & 0.43 & $\mathbf{0 . 9 0}$ & \\
8 & & & $\mathbf{0 . 8 0}$ & & 0.41 & $\mathbf{0 . 8 9}$ & \\
9 & & & $\mathbf{0 . 7 8}$ & & 0.48 & 0.86 & \\
\hline
\end{tabular}

(b)

Table 4: Fraction of correct commands for the DOMOTICA-4 database for each speaker in function of the number of blocks in the training set. Each column contains the results for one user. (a) Baseline system (b) Proposed system

DOMOTICA - 4 database. For this database the proposed system performs a bit worse for speaker 2 and the results are comparable for speakers 5 and 6 . For speakers 1, 3, 4 and 7 the proposed system performs better for all training set sizes. On average it can be concluded that the proposed system outperforms the baseline system. This conclusion is however a bit less clear than for the GRABO and PATCOR databases.

\section{Future work}

In section 3 it was mentioned that labels are added that ensure that all labels in a family sum to one. This label is one if none of the other labels are active and zero otherwise. However adding these labels is not really optimal because this introduces some redundancy in the labels. The only case were none of the labels in a family are active is when the parent label (the label above the family in the hierarchy) is not active. This means that the added label is always the complement of the parent label. This redundancy can be removed by taking the parent label into account when considering a family. This way all the levels are linked through the parents making it difficult to calculate the derivative. An efficient method for deriving the cost function in this case must be developed.

\section{Conclusions}

In this paper a new cost function is proposed for learning the semantic dictionary based on the multinomial likelihood function. This cost function takes the mutual exclusivity of the labels into account. Based on this cost function an updating scheme is proposed. The proposed system is tested and compared to the baseline system and the results show a significant improvement over the baseline system in almost all scenarios. 


\section{References}

[1] T. Kawahara, C.-H. Lee, and B.-H. Juang, "Flexible speech understanding based on combined key-phrase detection and verification," Speech and Audio Processing, IEEE Transactions on, vol. 6, no. 6 , pp. $558-568,1998$.

[2] R. Kuhn, J.-C. Junqua, P. Nguyen, and N. Niedzielski, "Rapid speaker adaptation in eigenvoice space," Speech and Audio Processing, IEEE Transactions on, vol. 8, no. 6, pp. 695-707, 2000.

[3] H. Christensen, S. Cunningham, C. Fox, P. Green, and T. Hain, "A comparative study of adaptive, automatic recognition of disordered speech." in INTERSPEECH, 2012.

[4] K. T. Mengistu and F. Rudzicz, "Comparing humans and automatic speech recognition systems in recognizing dysarthric speech," in Advances in Artificial Intelligence. Springer, 2011, pp. 291-300.

[5] J. F. Gemmeke, J. Van De Loo, G. De Pauw, J. Driesen, H. V. hamme, and W. Daelemans, "A self-learning assistive vocal interface based on vocabulary learning and grammar induction," in INTERSPEECH, 2012.

[6] B. Ons, N. Tessema, J. Van De Loo, and J. F. Gemmeke, "A self learning vocal interface for speech-impaired users," SLPAT 2013 , p. $73,2013$.

[7] J. Gemmeke, S. Sehgal, and S. Cunningham, "Fast vocabulary learning for disordered speech vocal interfaces," in Spoken Language Technology Workshop (SLT). IEEE, 2014.

[8] C. Févotte and A. T. Cemgil, "Nonnegative matrix factorizations as probabilistic inference in composite models," in Proc. EUSIPCO, vol. 47, 2009, pp. 1913-1917.

[9] H. Van hamme, "HAC-models: a novel approach to continuous speech recognition," in Proceedings Interspeech, ISCA, 2008.

[10] Y.-Y. Wang and A. Acero, "Rapid development of spoken language understanding grammars," Speech Communication, vol. 48, no. 3, pp. 390-416, 2006

[11] D. D. Lee and H. S. Seung, "Algorithms for non-negative matrix factorization," in Advances in neural information processing systems, 2001, pp. 556-562.

[12] _ - "Learning the parts of objects by non-negative matrix factorization," Nature, vol. 401, no. 6755, pp. 788-791, 1999.

[13] J. Driesen, "Discovering words in speech using matrix factorization," KU Leuven, ESAT, 2012.

[14] N. Tessema, B. Ons, J. van de Loo, J. Gemmeke, G. De Pauw, W. Daelemans, and H. Van hamme, "Metadata for corpora patcor and domotica-2," Technical report KUL/ESAT/PSI/1303, KU Leuven, ESAT, Leuven, Belgium, 2013. [Online]. Available: https://irias.kuleuven.be/handle/123456789/410287 